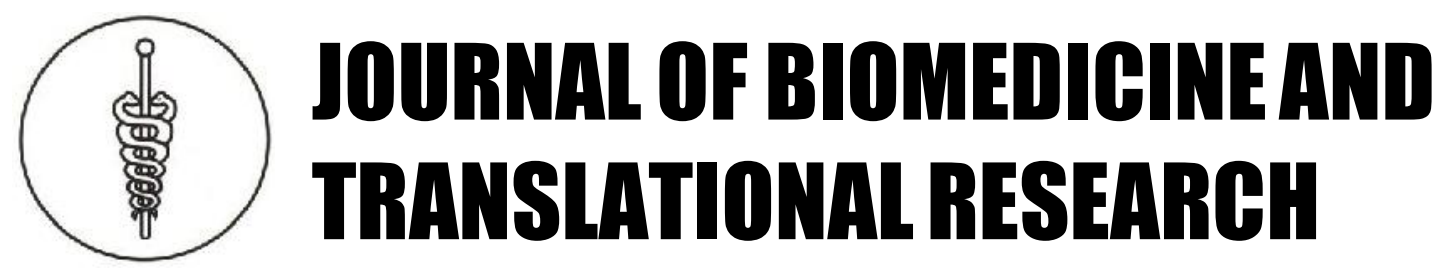

Copyright@2015 by Faculty of Medicine Diponegoro University and Indonesian Doctor Association, Central Java Region

\title{
JAK2 V617F Analysis in Indonesian Myeloproliferative Neoplasms Patients
}

\author{
Fanti Saktini $^{1,2}$, Santosa $^{3}$, Sultana MH Faradz ${ }^{2}$ \\ ${ }^{1}$ Histology Department, Faculty of Medicine, Diponegoro University \\ ${ }^{2}$ Medical Hematology-Oncology Division, Dr.Kariadi General Hospital/Faculty of Medicine, Diponegoro University \\ ${ }^{3}$ Center for Biomedical Research, Faculty of Medicine, Diponegoro University
}

\section{Article info}

History :

Received 17 March 2015

Accepted 27 March 2015

Available 30 December 2015

\begin{abstract}
Background: Three subtypes of myeloproliferative neoplasms (MPNs): Polycythemia vera (PV), essential thrombocythemia (ET), and primary myelofibrosis (PMF) showed overlapping phenotype. There has been no specific cytogenetic marker identified in these subtypes. JAK2 gene has a critical role in the pathogenesis of MPNs. Similar mutation, namely JAK2 V617F mutation, was found in PV, ET and PMF.

Objective :This study was done to define the prevalence of JAK2 V617F mutation in Indonesian MPNs patients.

Methods : This is a cross-sectional study of 187 patients who were referred to Center for Biomedical Research (CEBIOR) for JAK2 V617F mutation analysis. The study period was November 2010 until November 2015. It was analysed using Amplification Refractory Mutation System Polymerase Chain Reaction (ARMS-PCR) from peripheral blood vein. Clinical data were secondary data retrieved from hospital medical records.

Results : The prevalence of JAK2 V617F mutation in Indonesian MPNs patients was 107 out of 188 patients $(56.92 \%)$. Mutation prevalence distribution for each subtypes were 43 out of $70(61.43 \%)$ in PV, 25 out of $53(47.17 \%)$ in ET, 4 out of $6(66.67 \%)$ in PMF, whereas in unspecified MPN/MPD/MDS 35 out of $59(59.32 \%)$.

Conclusion : The prevalence of JAK2 V617F mutation was found comparable with previous studies in Indian MPNs. JAK2 V617F testing should be incorporated in the management therapy of MPNs in Indonesia.
\end{abstract}

Keywords: ARMS-PCR, JAK2 V617F, ET, MPN, PMF, PV

\section{INTRODUCTION}

Myeloid malignancies are stem cell-derived and clonal disorders, consist of three wide-ranging clinicopathologic categories: acute myeloid leukemia (AML), myelodysplastic syndrome (MDS) and myeloproliferative neoplasm (MPNs). MPNs were first acknowledged by William Damashek in $1951^{1}$ The classic MPNs were grouped into four subtypes, namely polycythemia vera (PV), essential thrombocythemia (ET), primary myelofibrosis (PMF) and chronic myelogenous leukemia (CML).
They were initially grouped based on their common phenotype of proliferation. Because of their similarities in increasing mature peripheral blood cells and overlapping phenotype, diagnosis has been difficult to be established in the past. It was believed that it came from similar unknown mechanism. ${ }^{1}$ The North American Association of Central Cancer Registries (NAACCR) stated that the age-adjusted incidence rate was 2.1 per 100,000 in $2001-2003 .^{2}$ For each disease subtype, an earlier study in Sweden reported the annual prevalence per 100,000 inhabitants for PV, ET, and PMF were $2-2.8 ; 1.5$; and 0.4 respectively. ${ }^{3,4}$ 
The important issues in the course of MPNs are thrombosis, hemorrhage, evolution to post-polycythemic or post-thrombocythemic myelofibrosis and AML transformation. ${ }^{2}$ Thrombosis and bleeding are the leading causes of morbidity in MPNs. ${ }^{5}$ In one-third of MPNs patients, early vascular events constitutes first disease manifestation. ${ }^{6}$ Even though thrombosis is the most frequent complication in MPNs, but bleeding is more observed in ET. ${ }^{7}$

The understanding of the molecular pathogenesis of myeloid malignancies has fundamentally derived from the identification of $\mathrm{t}(9 ; 22)(\mathrm{q} 34 ; \mathrm{q} 11)$ or Philadelphia chromosome in CML. However, in many patients with MPNs, no specific abnormality has been identified to date. The frequency of cytogenetic abnormalities in the Philadelphia-negative MPNs varies from approximately $40 \%$ in PMF to $3 \%$ in $\mathrm{ET}^{8}$. The spectrum of aberrations is heterogeneous, ranging from gains and losses of genetic material to structural changes including unbalanced translocations. ${ }^{9}$ The role of cytogenetic abnormalities as a prognostic marker in PMF has been suggested, both at the time of diagnosis and later during disease course. ${ }^{10}$

In molecular level, Philadelphia chromosome is derived from two genes fusion, BCR-ABL on the $22 q-$ and the reciprocal $\mathrm{ABL}-\mathrm{BCR}$ on $9 \mathrm{q}-$, resulting a chimeric gene BCR-ABL. The identification of the BCR-ABL gene and consequent protein led to the production of small-molecule drugs, proposed to hinder BCR-ABL tyrosine kinase activation by competitive binding at the ATP-binding site: Imatinib mesylate (IM). ${ }^{11}$ IM turn out to be the first drug of choice in chronic phase CML, as a result of its high efficacy, low toxicity and capacity to preserve strong hematological and cytogenetic responses. $^{11}$

Several recent discoveries have identified a central role of protein tyrosine kinase (PTK) in the pathogenesis of MPNs. Several groups reported the discovery of JAK2 V617F mutation in early 2005. ${ }^{12-14}$ Baxter et al (2005) found a single base substitution, guanine to thymine change at 1849 , which resulted in the change of valine to phenylalanine in exon 14 of the pseudokinase domain of tyrosine kinase JAK2 (Janus Kinase 2) gene in $97 \% \mathrm{PV}$, $57 \%$ ET and $50 \% \mathrm{PMF}^{12}$ This mutation results in a gain of function due to the constitutive activation of tyrosine kinase-dependent cellular signaling pathways, particularly of the JAK-STAT (Signal Transducers and Activators of Transcription). The pathway is principal in regulation of cell proliferation, differentiation and apoptosis in.$^{14}$

JAK2 V617F mutation as a common genetic abnormality in PV, ET, and PMF had pointed the possibility of using tyrosine kinase as a valid therapy target. The use of drug that targets the tyrosine kinases is expected to follow the efficacy of IM and other tyrosine kinase inhibitors in CML. ${ }^{2,7}$

MPNs patients who carried JAK2 V617F mutation have been associated with older age at diagnosis (ET and PMF), ${ }^{15}$ higher hemoglobin level (ET and PMF), ${ }^{16}$ leukocytosis (ET and PMF), ${ }^{15}$ lower platelet count (ET), larger spleen size (PV, ET and PMF), ${ }^{17}$ the need for spelenectomy, ${ }^{17}$ and leukemic transformation ${ }^{17}$. Patients with mutation have been associated with shorter survival in PMF, but less likely to require blood transfusion. ${ }^{18}$
Dunlap et al studied the correlation between cytogenetic abnormalities with disease stage and JAK2 V617F status in MPNs and MDS/MPNs patients. Cytogenetic data were available in ninety-seven out of 179 cases $(54,19 \%)$. JAK2 V617F positive group showed higher frequency of chromosomal abnormalities (51\% vs. $27 \%$ ). In JAK2 V617F positive group, the commonest abnormalities were found in chromosome 9 , chromosome 7 , chromosome $20 \mathrm{q}$, while $13 \mathrm{q}$ and trisomy 21 were frequent in $J A K 2 \mathrm{~V} 617 \mathrm{~F}$ negative group. Chromosome 7 and complex abnormalities were associated with blastic transformation. ${ }^{19}$

This study aim was to define the prevalence of JAK2 V617F mutation in Semarang MPNs patients.

\section{METHODS}

This is a cross-sectional study of 187 patients who were referred to Center for Biomedical Research (CEBIOR) for JAK2 V617F mutation analysis. Myeloproliferative neoplasm's consisted of PV, ET, PMF and/or MPNs unclassified subtypes. The diagnosis was determined by the reffering clinician according to clinical symptoms and signs, and other supporting data. The study period was November 2010 until November 2015.

DNA was extracted from peripheral blood using the salting out method at the Center for Biomedical Research (CEBIOR) Faculty of Medicine, Diponegoro University (FMDU). JAK2 V617F mutation was obtained using the Amplification Refractory Mutation System (ARMS-PCR) according to Baxter et al with some minor modifications. ${ }^{12}$ The principle of the method is using one common reverse primer and two forward primers. One forward primer would be specifically paired to the mutant JAK2 (if the patient carry the mutation) and the other forward primer would be paired with the wild-type (if the patient did not carry the mutation). The third base from 3' end was intentionally mismatched to maximize the allele specificity. The appearance of two bands at $364 \mathrm{bp}$ and $203 \mathrm{bp}$ is determined as positive, and single band at $364 \mathrm{bp}$ as negative. The $364 \mathrm{bp}$ band also acted as internal control for every sample (See Figure 1). DNA Sequencing to ascertain the presence of $\mathrm{G}$ to $\mathrm{T}$ nucleotide substitution at position 1849 was done in The Agency for The Assessment and Application of Technology/Badan Pengkajian dan Penerapan (BPPT) in Jakarta (See Figure 2).

Patient's clinical data, the result of routine hematology examination, bone marrow smear analysis, and/or relevant findings were retrieved from hospital medical record and summarized in a case report form for each subject.

The data were tested for mutation status difference in proportions using chi-square test. Unpaired $t$-test was used to compare continuous variables between JAK2 $\mathrm{V} 617 \mathrm{~F}$ positive and negative groups such as age, and blood count, except for leukocytes.

The ethical clearance was approved by the Health Research Ethical Committee of MFDU/Dr. Kariadi Hospital, Semarang and Dr. Kariadi Hospital Research 
Review Board. Study subjects were provided with informed consent.

\section{RESULTS}

During the study period, there were 188 patients from around Central Java who were referred for JAK2 V617F mutation. (Table 1). There were 59 patients who were diagnosed as unspecified MPNs, myeloproliferative disease (MPD) or myelodysplastic syndrome (MDS), while there were 70 patients who were diagnosed as PV or having high hemoglobin level, 53 patients diagnosed as ET or showing thrombocytosis, and 6 diagnosed as myelofibrosis (See Table 1). Among the four groups, only the ET/Thrombocytosis group showed lower proportion of $J A K 2$ V617F-positive mutation.

Table 1. Referral diagnosis for JAK2 V617F analysis

\begin{tabular}{lccc}
\hline $\begin{array}{c}\text { MPNs Referral } \\
\text { Diagnosis }\end{array}$ & $\begin{array}{c}J A K 2 \\
(+)\end{array}$ & $\begin{array}{c}\text { V617F } \\
(-)\end{array}$ & $P$ \\
& $\mathrm{n}(\%)$ & $\mathrm{n}(\%)$ & \\
\hline MPN/MPD/MDS & $35(59.32)$ & $24(40.68)$ & 0.39 \\
PV & $43(61.43)$ & $27(38.57)$ & \\
ET/Thrombocytosis & $25(47.17)$ & $28(52.83)$ & \\
Myclofibrosis & $4(66.67)$ & $2(33.33)$ & \\
\cline { 2 - 4 } & $107(56.92)$ & $81(43.08)$ & \\
\hline
\end{tabular}

Other than diagnosis in MPNs, several of the study subjects had other diagnosis in hematology or vascular disease (See Table 2). These conditions showed a possibility that they also experienced vascular events, especially thrombosis that was related to their MPNs diagnosis and JAK2 V617F mutational status. Diagnosis involving other systems showed that elevation of blood count (in PV and ET) might be an incidental finding that was found during the diagnosis work-up, not solely derived from symptoms and signs of MPNs

Table 2. Accompanying hematology and/or vascular event (thrombosis) diagnosis

\begin{tabular}{lccc}
\hline Diagnosis & $n$ & \multicolumn{2}{c}{$J A K 2$ V617F } \\
& & Positive & Negative \\
\hline Aplastic anemia & 1 & 1 & 0 \\
Anemia & 1 & 0 & 1 \\
Trombocytopenia & 3 & 2 & 1 \\
Pancytopenia & 1 & 0 & 1 \\
Leukocytosis & 5 & 4 & 1 \\
Leukemia & 2 & 2 & 0 \\
Acute Myeloid Leukemia, Non Haemorrhagic Stroke & 1 & 0 & 1 \\
Combination of Anemia, Leukocytosis, Splenomegaly & 3 & 2 & 1 \\
CML & 1 & 0 & 1 \\
Splenomegaly & 3 & 2 & 1 \\
Non Haemorhagic Stroke & 1 & 1 & 0 \\
Stroke & 1 & 0 & 1 \\
Tischemic Heart Disease & 1 & 0 & 1 \\
Acute Myocardial Infarction & 1 & 1 & 0 \\
Old Myocardial Infarction & 2 & 2 & 0 \\
Acute Coronary Syndrome & 1 & 0 & 1 \\
Pperipheral Artery Disease & 1 & 0 & 1 \\
Deep Vein Thrombosis & 2 & 0 & 2 \\
Thallasemia & 1 & 0 & 1 \\
\hline Total & 32 & 17 & 15
\end{tabular}

Table 3. Accompanying diagnosis involving other systems

\begin{tabular}{|c|c|c|c|}
\hline \multirow[t]{2}{*}{. } & \multicolumn{3}{|c|}{$J A K 2$ V617Fmutation } \\
\hline & $n$ & Fositive & Negativa \\
\hline $\begin{array}{l}\text { Grace II aypertersior, Multip.e Renal cysts, renal insufficiency, } \\
\text { hypoa.bumn lung infiltrate. pyuria, hematura. }\end{array}$ & 1 & 1 & 0 \\
\hline $\begin{array}{l}\text { Grace ll aypertersior, chronic kidney disease stage IV, renal } \\
\text { insıfficiency, hypoalhumn, lung infiltrate, pyivia, hematura }\end{array}$ & 1 & 1 & 0 \\
\hline Rught ovanan cyst & 1 & 1 & 0 \\
\hline Wide stomattit, eeophegeal curdidesie, pleuropreumonia, zastric uicer & 1 & 0 & 1 \\
\hline Melena & 1 & 1 & 0 \\
\hline Gcut Arthritis & 1 & 1 & 0 \\
\hline Duplex rhinosinusitis maxilaris & 1 & 0 & 1 \\
\hline Wnumd dehissence & 1 & 0 & 1 \\
\hline Post-cranictcr:y & 1 & 1 & 0 \\
\hline Fever & 2 & 1 & 1 \\
\hline Liver chirosis & 1 & 1 & 0 \\
\hline Diabetic ulser & 1 & 0 & 1 \\
\hline Vertigo & 1 & 0 & 1 \\
\hline Focal Nodular Hyp:rpla sia & 1 & 1 & 0 \\
\hline Post hematoma explcration & 1 & 1 & 0 \\
\hline DM, Ikterus & 1 & 1 & 0 \\
\hline Herriparesis & 1 & 1 & 0 \\
\hline Urne retansion & 1 & 1 & 0 \\
\hline Space occupyinglesicn & 1 & 0 & 1 \\
\hline \multirow[t]{2}{*}{ Post-Odcntectomy } & 2 & 1 & 1 \\
\hline & 22 & 14 & 8 \\
\hline
\end{tabular}

Table 4 showed that positive cases were older than negative cases $(54.43 \pm 11.857$ vs. $49.01 \pm 16.351)$, although it was insignificant. In both sex groups, more positive cases were observed in the study subjects. Blood count at diagnosis showed that positive cases had higher hemoglobin and hematocrite values. In the contrary, leukocyte and platelet counts were lower in positive group. Four JAK2 V617F mutation positive subjects (3 PV and 1 ET) was confirmed with sequencing, and showed the present of $\mathrm{G}$ to $\mathrm{T}$ nucleotide substitution (Figure 2).

Table 4. Demographic characteristics

\begin{tabular}{cccc}
\hline & \multicolumn{2}{c}{ JAK2 V617F mutation } & \multirow{2}{*}{$P$} \\
\cline { 2 - 3 } & Positive & Negative & \\
\hline Age (year) & $54.43 \pm 11.857$ & $49.01 \pm 16.351$ & 0.09 \\
Sex & $62 / 113$ & $45 / 75$ & 0.49 \\
Male & $62(54.86 \%)$ & $51(45.14)$ & \\
Female & $45(60 \%)$ & $35(40 \%)$ & \\
& & & \\
& & &
\end{tabular}

Table 5. Blood count

\begin{tabular}{|c|c|c|c|c|c|}
\hline & \multicolumn{4}{|c|}{$J A K 2$ V617F mulation } & \multirow{2}{*}{$p$} \\
\hline & $N$ & $\begin{array}{l}\text { Posilive } \\
\text { mean } \pm S D\end{array}$ & $n$ & $\begin{array}{l}\text { Negative } \\
\text { mean=SD }\end{array}$ & \\
\hline Hemoglobin (g\%) & 22 & $15.6 . \pm 5.20$ & 11 & $12.0 \pm 5.66$ & 0.79 \\
\hline Hematocrite (\%) & 20 & $44.9 \pm 18.03$ & 11 & $34.4=16.71$ & 0.12 \\
\hline Leukocyte $\left(/ \mathrm{mm}^{3}\right)^{2}$ & 20 & $23.0 \perp 13.16$ & 9 & $49.3-90.74$ & 0.41 \\
\hline Platelet $\left(10^{3} / \mathrm{mm}^{3}\right)$ & 20 & $798.1 \pm 533.08$ & 11 & $1,038.6 \pm 183.65$ & 0.22 \\
\hline
\end{tabular}

Comparison was tested using unpaired t-test, except for leukocyte (Fischer's exact test) 


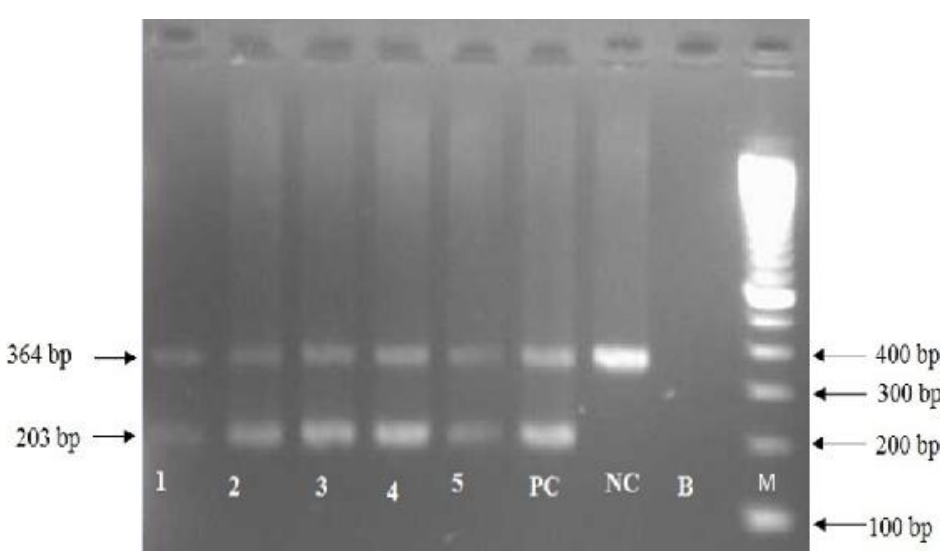

Figure 1. JAK2 V617F mutation detection using ARMSPCR. Lane 1 to 5 represented subjects number 12/F/11 to 16/F/11). Upper band at $364 \mathrm{bp}$ were the internal PCR control which appeared in every sample tested. Lower band at $203 \mathrm{bp}$ indicated the presence of mutation. Positive control (PC) was the positive result from previous PCR, negative control (NC) came from nonMPNs individual, (B) is blank, (M) 100 bp marker ladder.

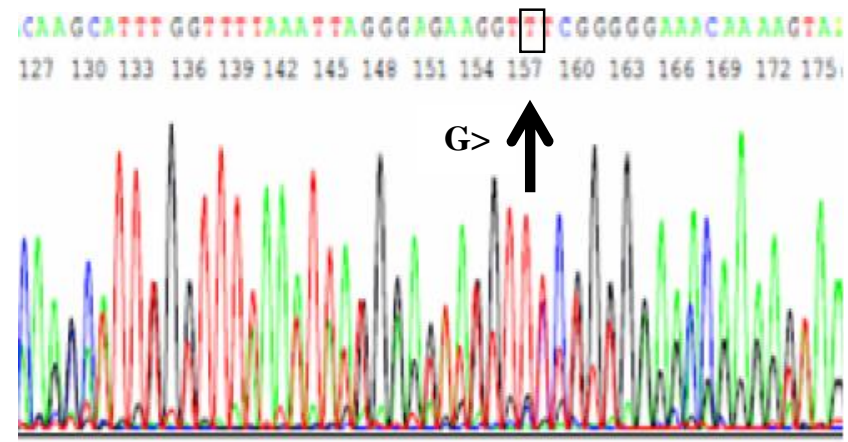

Figure 2. Sequencing profile of a subject (07/F/11) whose PCR positive for JAK2 V617F mutation using forward primer. Arrow indicated the presence of mutation c. $1849 \mathrm{G}>\mathrm{T}$ in exon 14 of JAK2 gene.

\section{DISCUSSION}

This study results supported the fact that $J A K 2 \mathrm{~V} 617 \mathrm{~F}$ mutations were found in majority of MPNs patients $(56.92 \%)$. A study in India reported $68 \%$ of MPNs patients harbored this mutation. ${ }^{20}$ The distribution of $J A K 2 \mathrm{~V} 617 \mathrm{~F}$ mutation in the disease subtypes were similar with other reports in Caucasian, ${ }^{12,13,17}$ except for PV. This was possibly caused by different cut-off point that has been used by reffering clinicians.

Although the routine blood count values were not significantly different between the two groups, the profile that was observed in our findings was similar with other studies. The conclusion of a meta-analysis measuring the effect of $J A K 2 \mathrm{~V} 617 \mathrm{~F}$ on thrombotic risk in 492 studies suggested that $J A K 2 \mathrm{~V} 617 \mathrm{~F}$-positive patients were older at diagnosis, had higher hemoglobin, but lower thrombocyte counts. ${ }^{21}$ The most possible mechanism underlying these observations was that JAK2 V617F mutation caused hypersensitivity to cytokine stimulation. $^{13}$
A study which correlated JAK2 mutation status, hemostatic risk factors and thrombophilic factors in ET patients stated that JAK2 V617F mutation have been associated with older age at diagnosis (ET and PMF), higher hemoglobin level (ET and PMF), ${ }^{16}$ leukocytosis $(\mathrm{ET}$ and $\mathrm{PMF}){ }^{15}$ lower platelet count $(\mathrm{ET})^{17}$. In addition, JAK2 $\mathrm{V} 617 \mathrm{~F}$ mutational status predicts progression to large splenomegaly and leukemic transformation in primary myelofibrosis in PV, ET and PMF. ${ }^{17}$ Shorter survival in PMF patients have been associated with the JAK2 V617F mutation, but less likely to require blood transfusion. ${ }^{18}$ High proportion of $J A K 2$ V617F mutation which was found in our study subjects should be carefully followed-up by the reffering clinicians, in concern of the disease course related to positive results.

Among patients with hemoglobin level above normal cut-offs, the presence of JAK2 V617F mutation allows the diagnosis of PV in $>95 \%$ cases. Less than $2 \%$ of these patients might carry JAK2 exon 12 mutations. ${ }^{22}$ Further study should elucidate the presence of other $J A K 2$ mutations in JAK2 V617F negative patients.

The demonstration of $J A K 2 \mathrm{~V} 617 \mathrm{~F}$ mutation in patients with thrombocyte count $<600.000 / \mathrm{dL}$ (according to 2001 WHO classification system), ${ }^{23}$ supported the use of lower cut-off of thrombocyte count for ET diagnosis in the 2008 revision (changed to > $450.000 / \mathrm{dL}$ ) and might help the exclusion of reactive thrombocytosis. ${ }^{16,24}$ However, JAK2 V617F mutation was found only in $47.17 \%$ ET and $66.67 \%$ PMF cases. Thus, bone marrow smear analysis remains as an important diagnosis tool of ET, since bone marrow appearance in ET patients is distinct from typical PV or $\mathrm{PMF}$, and vice versa for $\mathrm{PMF}^{25}$

The use of peripheral blood, as in our study, has been reported as objective and made the JAK2 mutations screening become more accessible and practical in order to study patients suspected having MPNs, compare to invasive test like bone marrow examination or expertisedependent test such as direct red cell mass measurement. ${ }^{28}$ However, this molecular testing alone could not outweigh the important baseline information yielded from bone marrow histology and cytogenetic analysis.

Results of studies in familial MPNs about increased risk of first-degree relatives might improve the information that the $J A K 2 \mathrm{~V} 617 \mathrm{~F}$ testing should be provided for families of MPNs patients.

However, there were some limitations of the study. First, only JAK2 V617F mutation was studied. Despite the fact that the mutation is the commonest in MPNs, a large number of other mutations have been discovered and it has not been understood yet how is the hierarchy in this genetic complexity of MPNs. Second, the diagnosis of MPNs subtypes was made without incorporating red cell mass, serum Epo level or EEC (the complete 2008 WHO diagnostic criteria) although the remaining criteria were sufficient to establish the diagnosis. Third, the research setting was at only one center, given that the prevalence of MPNs is 2,1 per 
100.000 individuals a multicenter study might yield higher number of participants. ${ }^{2}$

\section{CONCLUSION}

Supported by the high incidence of JAK2 V617F mutation in our results, which were comparable with studies for in Indian and Caucasian we would recommend the integration of $J A K 2 \mathrm{~V} 617 \mathrm{~F}$ mutation testing in the diagnosis and management of MPNs cases in Indonesia.

\section{SUGGESTIONS}

Further studies should be conducted to explore the association of cytogenetic and JAK2 V617F mutation, clinical profiles, complications and prognosis in MPNs. As well as the exploration of other mutations that involved in the pathogenesis of MPNs. The main goal of the follow-up studies are to establish $J A K 2$ inhibitors for $J A K 2 \mathrm{~V} 617 \mathrm{~F}$-positive patients in clinical practice.

\section{ACKNOWLEDGEMENTS}

This research was funded by RISBIN IPTEKDOK 2011 Grant, Ministry of Health. FS is a recipient of Beasiswa Unggulan scholarship from Ministry of Education. The authors would like to thank the President of Diponegoro University; Dean of MFDU, Director \& Staffs of Center for Biomedical Research MFDU, Director of Kariadi General Hospital (KGH), Doctors \& Staffs of Medical Hematology-Oncology Division KGH, in Semarang, and staffs of Genetic Laboratory The Agency for The Assessment and Application of Technology/Badan Pengkajian dan Penerapan (BPPT) in Serpong. The authors also thanked Dr. dr. Hardian for his valuable advice in statistic analysis.

\section{REFERENCES}

1. Wadleigh $\mathrm{M}$, Tefferi A. Classification and diagnosis of myeloproliferative neoplasms according to the 2008 World Health Organization criteria. Int J Hematol 2010; 91: 174-9.

2. Vannucchi AM, Guglielmelli P, Tefferi A. Advances in understanding and management of myeloproliferative neoplasms. CA Cancer J Clin 2009; 59: 171-91.

3. Johansson P. Epidemiology of the myeloproliferative disorders polycythemia vera and essential thrombocythemia. Semin Thromb Hemost 2006; 32: 171-3.

4. Kutti J, Ridell B. Epidemiology of the myeloproliferative disorders: essential thrombocythaemia, polycythaemia vera and idiopathic myelofibrosis. Pathol Biol (Paris) 2001; 49: 164-6.

5. Vianello F, Battisti A, Cella G, Marchetti M, Falanga A. Defining the Thrombotic Risk in Patients with Myeloproliferative Neoplasms. TheScientificWorldJOURNAL 2011; 11: 1131-7.

6. Landolfi R, Di Gennaro L. Pathophysiology of thrombosis in myeloproliferative neoplasms. Haematologica 2011; 96: 183-6.
7. Passamonti F, Maffioli M, Caramazza D, Cazzola M. Myeloproliferative neoplasms: from $J A K 2$ mutations discovery to JAK2 inhibitor therapies. Oncotarget 2011; 2: 485-90.

8. Bacher U, Schnittger S, Kern W, Weiss T, Haferlach T, Haferlach C. Distribution of cytogenetic abnormalities in myelodysplastic syndromes, Philadelphia negative myeloproliferative neoplasms, and the overlap MDS/MPN category. Ann Hematol 2009; 88: 1207-13.

9. Reilly JT. Pathogenetic insight and prognostic information from standard and molecular cytogenetic studies in the BCR-ABL-negative myeloproliferative neoplasms (MPNs). Leukemia 2008; 22: 1818-27.

10. Tam CS, Abruzzo LV, Lin KI, Cortes J, Lynn A, Keating MJ et al. The role of cytogenetic abnormalities as a prognostic marker in primary myelofibrosis: applicability at the time of diagnosis and later during disease course. Blood 2009; 113: 4171-8

11. Gora-Tybor J, Robak T. Targeted drugs in chronic myeloid leukemia. Curr Med Chem 2008; 15: 3036-51.

12. Baxter EJ, Scott LM, Campbell PJ, East C, Fourouclas N, Swanton $\mathrm{S}$ et al. Acquired mutation of the tyrosine kinase JAK2 in human myeloproliferative disorders. Lancet 2005; 365: 1054-61.

13. Levine RL, Wadleigh M, Cools J, Ebert BL, Wernig G, Huntly BJ et al. Activating mutation in the tyrosine kinase $J A K 2$ in polycythemia vera, essential thrombocythemia, and myeloid metaplasia with myelofibrosis. Cancer Cell 2005; 7: 387-97.

14. Cazzola M, Skoda R. Gain of function, loss of control - a molecular basis for chronic myeloproliferative disorders. Haematologica 2005; 90: 871-4.

15. Vannucchi AM, Antonioli E, Guglielmelli P, Rambaldi A, Barosi G, Marchioli R et al. Clinical profile of homozygous JAK2 $617 \mathrm{~V}>\mathrm{F}$ mutation in patients with polycythemia vera or essential thrombocythemia. Blood 2007; 110: 840-6.

16. Sokolowska B, Nowaczynska A, Bykowska K, Chocholska S, Wejksza K, Walter-Croneck A et al. JAK2 mutation status, hemostatic risk factors and thrombophilic factors in essential thrombocythemia (ET) patients. Folia Histochem Cytobiol 2011; 49: 267-71.

17. Barosi G, Bergamaschi G, Marchetti M, Vannucchi AM, Guglielmelli P, Antonioli E et al. JAK2 V617F mutational status predicts progression to large splenomegaly and leukemic transformation in primary myelofibrosis. Blood 2007; 110: 4030-6.

18. Campbell PJ, Griesshammer M, Dohner K, Dohner H, Kusec R, Hasselbalch $\mathrm{HC}$ et al. V617F mutation in JAK2 is associated with poorer survival in idiopathic myelofibrosis. Blood 2006; 107: 2098-100. 
19. Dunlap J, Kelemen K, Leeborg N, Braziel R, Olson S, Press $\mathrm{R}$ et al. Association of JAK2 mutation status and cytogenetic abnormalities in myeloproliferative neoplasms and myelodysplastic/myeloproliferative neoplasms. Am J Clin Pathol 2011; 135: 709-19.

20. Sazawal S, Bajaj J, Chikkara S, Jain S, Bhargava $\mathrm{R}$, Mahapatra $\mathrm{M}$ et al. Prevalence of JAK2 V617F mutation in Indian patients with chronic myeloproliferative disorders. Indian $\mathrm{J}$ Med Res 2010; 132: 423-7.

21. Ziakas PD. Effect of JAK2 V617F on thrombotic risk in patients with essential thrombocythemia: measuring the uncertain. Haematologica 2008; 93: 1412-4.

22. Zhan H, Spivak JL. The diagnosis and management of polycythemia vera, essential thrombocythemia, and primary myelofibrosis in the JAK2 V617F era. Clin Adv Hematol Oncol 2009; 7: 334-42.

23. Antonioli E, Guglielmelli P, Poli G, Bogani C, Pancrazzi A, Longo $G$ et al. Influence of JAK2V617F allele burden on phenotype in essential thrombocythemia. Haematologica 2008; 93: 41-8.

24. Schafer AI. Thrombocytosis. N Engl J Med 2004; 350: 1211-9.

25. Tefferi A, Vardiman JW. The diagnostic interface between histology and molecular tests in myeloproliferative disorders. Curr Opin Hematol 2007; 14: 115-22.

26. Tefferi A. The rise and fall of red cell mass measurement in polycythemia vera. Curr Hematol Rep 2005; 4: 213-7. 